survival estimates and Cox Proportional Hazards Regression were performed, adjusting for sex, histology, age group, region, and urban-rural residence. Rural residence was defined using Statistics Canada's "Rural and Small Town" definition of living in a region with a population of less than 10,000 people. Results: No significant difference between urban and rural residence was identified in crude KM survival estimates. Though not significant, 5 -year survival was generally better among rural residents than urban residents, except for rural residents with anaplastic astrocytoma. There remained no significant difference for Cox hazard ratios after adjustment for age, sex, or region. Conclusions: This is the first study to examine the effect of urban-rural residence on brain cancer survival. No significant differences for any histology were found, indicating equitable access to care for brain cancer patients in Canada, regardless of their location of residence.

PC3 - 179

doi:10.1017/cjn.2016.384

\section{Analysis of Glioblastoma Physical Characteristics in Patients Benefiting from Tumor Treating Electric Fields Therapy}

\section{P.P. San ${ }^{l}$, J. Timmons, E. Lok, K.D. Swanson, E.T. Wong ${ }^{I}$ Harvard Medical School/Beth Israel Deaconess Medical Center ewong@bidmc.harvard.edu psan@bidmc.harvard.edu}

Tumor treating electric fields (TTFields) are an established treatment for glioblastoma patients. But the anatomical and physical characteristics of the brain and tumor contributing to treatment efficacy are unknown. We contoured gross tumor volume (GTV) using ScanIP and measured tumor size according to the RANO criteria on 5 patients with recurrent glioblastomas who benefited from TTFields (cohort 1) and 5 who did not (cohort 2). Tumor surface area and geometric centroid distance (GCD) from the bilateral ventricles were computed. Wilcoxon rank sum test was used to compare these physical parameters between the two cohorts. The results showed that the respective median GTV was $11.6 \mathrm{~cm} 3$ and $38.1 \mathrm{~cm} 3(\mathrm{P}=0.0591)$, while the respective median GCD was $5.0 \mathrm{~cm}$ and $5.3 \mathrm{~cm}(\mathrm{P}=0.6761)$, in cohort 1 and 2 . The tumor size had a median of $8.2 \mathrm{~cm} 2$ in cohort 1 , as compared to a median of $53.9 \mathrm{~cm} 2$ in cohort $2(\mathrm{P}=0.0591)$. The surface area had a median of $56.2 \mathrm{~cm} 2$ in cohort 1 , as compared to a median of $214.0 \mathrm{~cm} 2$ in cohort $2(\mathrm{P}=0.4034)$. After removing an outlier from cohort 1 and another from cohort 2, the respective median GTV was $9.7 \mathrm{~cm} 3$ and $41.3 \mathrm{~cm} 3(\mathrm{P}=0.1003)$, and the respective median surface area was $46.5 \mathrm{~cm} 2$ and $236.0 \mathrm{~cm} 2 \quad(\mathrm{P}=0.0304)$. The respective median surface area/GCD was $13.3 \mathrm{~cm}$ and $38.9 \mathrm{~cm}$ $(\mathrm{P}=0.0304)$ and the respective median GTV/GCD was $2.7 \mathrm{~cm} 2$ and $8.5 \mathrm{~cm} 2(\mathrm{P}=0.0304)$. The data suggest that the ratio of surface area/GCD and GTV/GCD, which are proportional to the tissue capacitance, may be an important parameter for optimizing TTFields efficacy in the treatment of glioblastoma.

PC3 - 180

doi:10.1017/cjn.2016.385

Is Conventional and Perfusion MRI Useful in Predicting Histopathology Defined Percentage of Recurrence in High Grade Gliomas

T.B. Nguye, University of Ottawa, Ottawa, ON thnguyen@toh.on.ca
New enhancing lesions after surgery and chemoradiation for high grade glioma commonly contain variable proportions of tumor recurrence (TR), tissue necrosis and treatment related changes. Our purpose is to determine whether the pattern of contrast enhancement and perfusion MR parameters correlate with the percentage of TR in these lesions. Methods: We prospectively enrolled 30 patients with high grade gliomas who presented with a new enhancing lesion suspicious for tumor recurrence. Each patient underwent conventional MRI with DCE and DSC perfusion MRI. The pattern of enhancement was classified by a blinded neuroradiologist in 5 different categories (solid, focal nodular, peripheral rim, hazy, punctate). A hot spot region-of-interest analysis was performed for each parametric map (Ktrans, AUC, $\mathrm{Vp}$, corrected CBV). TR percentage was defined histopathologically. The lesions were categorized into predominant TR (=tumor $>70 \%$ ), predominant treatment related changes $(\mathrm{T}=<35 \%)$ and mixed lesions $(35 \%<\mathrm{T}=<70 \%)$. Differences between the groups were assessed via Kruskal-Wallis and MannWhitney U tests. Results: There were 32 lesions (4 predominantly treatment related lesions, 5 mixed lesions, 23 predominant tumor recurrence). There is no significant difference in the enhancement pattern between the three groups $(\mathrm{p}=0.18)$. Statistically significant difference was only seen for corrected CBV between the three groups $(\mathrm{p}=0.01)$, mainly between the mixed and predominant tumor groups. The rest of the perfusion parameters did not show a statistically significant difference between the groups $(p>0.05)$. Conclusion: Corrected CBV might be useful in predicting the proportion of tumor recurrence in post-treatment high grade gliomas.

PC3 - 190

doi:10.1017/cjn.2016.386

Impact of Functional Magnetic Resonance Imaging on Clinical Decision Making and Outcomes in Patients with Low Grade Gliomas

\author{
E. Kosteniuk ${ }^{I}$, J.C. Lau, J.F. Megyesi \\ ${ }^{I}$ School of Medicine and Dentistry, University of Western Ontario, \\ London, $O N$ \\ skosteniuk2018@meds.uwo.ca
}

This study aims to evaluate the impact of preoperative functional magnetic resonance imaging (fMRI) on low grade glioma (LGG) patients' outcomes and surgical planning. Methods In this retrospective matched cohort study of a single surgeon's patients, we are comparing two groups of LGG patients (WHO grade II) based on exposure to fMRI. Sixteen LGG patients who underwent fMRI were selected, and 32 control (non-fMRI exposed) patients are being selected through propensity score matching from a pool of 764 brain tumour patients. To assess the impact of fMRI data on clinicians' decision making process, neurosurgeons within a single centre are completing questionnaires regarding treatment options for each LGG fMRI patient based on clinical data and structural imaging before and after fMRI. Results Within the group of 16 LGG patients who have undergone fMRI studies over a 12-year period, most patients presented with seizures (81 percent), and most lesions were left-sided (81 percent) and frontal (75 percent). Patients underwent either craniotomy (50 percent), stereotactic biopsy ( 25 percent) or nonsurgically management ( 25 percent). In surgical patients, between presurgical assessment and eight week post-surgical follow-up, mean modified Rankin scale improved from 1.80 (sd 0.79) to 1.50 (sd 0.97). In our cohort, 5-year 